\title{
Generalized inequalities on warped product submanifolds in nearly trans-Sasakian manifolds
}

\section{Abdulqader Mustafa, Siraj Uddin* and Bernardine Renaldo Wong}

\section{"Correspondence:} siraj.ch@gmail.com Institute of Mathematical Sciences, Faculty of Science, University of Malaya, Kuala Lumpur, 50603, Malaysia

\begin{abstract}
In this paper, we study warped product submanifolds of nearly trans-Sasakian manifolds. The non-existence of warped product semi-slant submanifolds of type $N_{\theta} \times{ }_{f} N_{T}$ is shown, whereas some characterization and new geometric obstructions are obtained for the warped products of type $N_{T} \times_{f} N_{\theta}$. We establish two general inequalities for the squared norm of the second fundamental form. The first inequality generalizes derived inequalities for some contact metric manifolds (Kadri et al. in J. Korean Math. Soc. 42:1101-1110, 2005; Munteanu in Publ. Math. (Debr.) 66:75-120, 2005; Mustafa et al. in Taiwan. J. Math. 17:1473-1486, 2013; Uddin and Khan in J. Inequal. Appl. 2012:304, 2012), while by a new technique, the second inequality is constructed to express the relation between extrinsic invariant (second fundamental form) and intrinsic invariant (scalar curvatures). The equality cases are also discussed. MSC: 53C40; 53C42; 53C15
\end{abstract}

Keywords: warped products; almost contact manifold; nearly trans-Sasakian manifold; semi-slant submanifold; scalar curvature; isometric immersion; minimal immersion; $\mathrm{N}_{T}$-minimal immersion

\section{Introduction}

In a natural way, warped products appeared in differential geometry generalizing the class of Riemannian product manifolds to a much larger one, called warped product manifolds, which are applied in general relativity to model the standard space time, especially in the neighborhood of massive stars and black holes $[1,2]$. These manifolds were introduced by Bishop and O'Neill [3]. They defined warped products as follows: Let $N_{1}$ and $N_{2}$ be two Riemannian manifolds with Riemannian metrics $g_{1}$ and $g_{2}$, respectively, and let $f>0$ be a differentiable function on $N_{1}$. Consider the product manifold $N_{1} \times N_{2}$ with its projections $\pi_{1}: N_{1} \times N_{2} \rightarrow N_{1}$ and $\pi_{2}: N_{1} \times N_{2} \rightarrow N_{2}$. Then their warped product manifold $M=$ $N_{1} \times_{f} N_{2}$ is the Riemannian manifold $N_{1} \times N_{2}=\left(N_{1} \times N_{2}, g\right)$ equipped with the Riemannian structure such that

$$
\|X\|^{2}=\left\|\pi_{1 \star}(X)\right\|^{2}+\left(f \circ \pi_{1}\right)^{2}\left\|\pi_{2 \star}(X)\right\|^{2}
$$

for any vector field $X$ tangent to $M$, where $\star$ is the symbol for the tangent maps. A warped product manifold $M=N_{1} \times N_{2}$ is said to be trivial or simply Riemannian product if the

\section{黛 Springer}

(02014 Mustafa et al.; licensee Springer. This is an Open Access article distributed under the terms of the Creative Commons Attribution License (http://creativecommons.org/licenses/by/2.0), which permits unrestricted use, distribution, and reproduction in any medium, provided the original work is properly cited. 
warping function $f$ is constant. For the survey on warped products as Riemannian submanifolds, we refer to $[4,5]$.

A $(2 m+1)$-dimensional $C^{\infty}$ manifold $(\bar{M}, g, \phi, \xi, \eta)$ is said to have an almost contact structure if there exist on $\bar{M}$ a tensor field $\phi$ of type $(1,1)$, a vector field $\xi$, a 1 -form $\eta$ and a Riemannian metric $g$ satisfying [6]

$$
\begin{aligned}
& \phi^{2}=-I+\eta \otimes \xi, \quad \phi \xi=0, \quad \eta \circ \phi=0, \quad \eta(\xi)=1, \\
& \eta(X)=g(X, \xi), \quad g(\phi X, \phi Y)=g(X, Y)-\eta(X) \eta(Y),
\end{aligned}
$$

where $X$ and $Y$ are vector fields on $\bar{M}$ [7]. We shall use the symbol $\Gamma(T \bar{M})$ to denote the Lie algebra of vector fields on the manifold $\bar{M}$.

In the classification of almost contact structures, Chinea and Gonzalez [8] divided these structures into twelve well-known classes; one of the class that appears in this classification is denoted by $C_{1} \oplus C_{5} \oplus C_{6}$. According to their classification, an almost contact metric manifold is a nearly trans-Sasakian manifold if it belongs to this class. Another line of thought was developed by Gherghe [9] who introduced nearly trans-Sasakian structure of type $(\alpha, \beta)$, which generalizes trans-Sasakian structure in the same sense as nearly Sasakian generalizes Sasakian one. In this sense an almost contact metric structure $(\phi, \xi, \eta, g)$ on $\bar{M}$ is called a nearly trans-Sasakian structure if

$$
\begin{aligned}
\left(\bar{\nabla}_{X} \phi\right) Y+\left(\bar{\nabla}_{Y} \phi\right) X= & \alpha(2 g(X, Y) \xi-\eta(Y) X-\eta(X) Y) \\
& -\beta(\eta(Y) \phi X+\eta(X) \phi Y)
\end{aligned}
$$

for any $X, Y \in \Gamma(T \bar{M})$. Moreover, nearly trans-Sasakian of type $(\alpha, \beta)$ is nearly-Sasakian, or nearly Kenmotsu, or nearly cosymplectic accordingly as $\beta=0$ or $\alpha=0$ or $\alpha=\beta=0$.

Kim et al. [10] initiated the study of semi-invariant submanifolds of nearly transSasakian manifolds and obtained many results on the extrinsic geometric aspects of these submanifolds, whereas the slant submanifolds were studied in the setting of nearly transSasakian manifolds by Al-Solamy and Khan [11]. Recently, we have initiated the study of CR-warped product in nearly trans-Sasakian manifolds [12]. In the present paper, we consider a warped product of proper slant and invariant submanifolds of nearly trans-Sasakian manifolds, called warped product semi-slant submanifolds. The paper is organized as follows. Section 2 is devoted to providing the basic definitions and formulas which are useful to the next section. In Section 3, general and special non-existence results are proved for warped products. In the case of existence of warped products, the necessary lemmas for the two inequalities and some geometric obstructions are obtained. In Section 4, a general inequality which generalizes the obtained inequalities in [12-15] is established. In Section 5, we develop a new technique to construct a general inequality for the second fundamental form in terms of the scalar curvatures of submanifolds and the warping function.

\section{Preliminaries}

Let $M$ be an $n$-dimensional Riemannian manifold isometrically immersed in a Riemannian manifold $\bar{M}$. Then the Gauss and Weingarten formulas are respectively given by

$$
\bar{\nabla}_{X} Y=\nabla_{X} Y+h(X, Y)
$$


and

$$
\bar{\nabla}_{X} N=-A_{N} X+\nabla_{X}^{\perp} N
$$

for all $X, Y \in \Gamma(T M)$, where $\nabla$ is the induced Riemannian connection on $M, N$ is a vector field normal to $\bar{M}, h$ is the second fundamental form of $M, \nabla^{\perp}$ is the normal connection in the normal bundle $T^{\perp} M$ and $A_{N}$ is the shape operator of the second fundamental form. They are related as

$$
g\left(A_{N} X, Y\right)=g(h(X, Y), N)
$$

where $g$ denotes the Riemannian metric on $\bar{M}$ as well as the metric induced on $M$. For any $X \in \Gamma(T M)$, we decompose $\phi X$ as follows:

$$
\phi X=P X+F X
$$

where $P X$ and $F X$ are the tangential and normal components of $\phi X$, respectively.

For a submanifold $M$ of an almost contact manifold $\bar{M}$, if $F$ is identically zero then $M$ is invariant, and if $P$ is identically zero then $M$ is anti-invariant.

For the orthonormal basis $\left\{e_{1}, \ldots, e_{n}\right\}$ of the tangent space $T_{x} M$, the mean curvature vector $\vec{H}(x)$ is given by

$$
\vec{H}(x)=\frac{1}{n} \sum_{i=1}^{n} h\left(e_{i}, e_{i}\right),
$$

where $n=\operatorname{dim}(M)$. The submanifold $M$ is totally geodesic in $\bar{M}$ if $h=0$, and minimal if $H=0$. If $h(X, Y)=g(X, Y) H$ for all $X, Y \in \Gamma(T M)$, then $M$ is totally umbilical.

Let $(M, g)$ be a submanifold of a Riemannian manifold $\bar{M}$ equipped with a Riemannian metric $g$. The equation of Gauss is given by

$$
\begin{aligned}
R(X, Y, Z, W)= & \bar{R}(X, Y, Z, W)+g(h(X, W), h(Y, Z)) \\
& -g(h(X, Z), h(Y, W))
\end{aligned}
$$

for all $X, Y, Z, W \in \Gamma(T M)$, where $\bar{R}$ and $R$ are the curvature tensors of $\bar{M}$ and $M$, respectively, and $h$ is the second fundamental form.

Definition 2.1 [16] An immersion $\varphi: N_{1} \times_{f} N_{2} \rightarrow \bar{M}$ is called $N_{i}$-totally geodesic if the partial second fundamental form $h_{i}$ vanishes identically. It is called $N_{i}$-minimal if the partial mean curvature vector $\vec{H}_{i}$ vanishes for $i=1,2$.

The scalar curvature $\tau(x)$ of $M$ is defined by

$$
\tau(x)=\sum_{1 \leq i<j \leq n} K\left(e_{i} \wedge e_{j}\right),
$$

where $K\left(e_{i} \wedge e_{j}\right)$ is the sectional curvature of the plane section spanned by $e_{i}$ and $e_{j}$ at $x \in M$. Let $\Pi_{k}$ be a $k$-plane section of $T_{x} M$, and let $\left\{e_{1}, \ldots, e_{k}\right\}$ be any orthonormal basis 
of $\Pi_{k}$. The scalar curvature $\tau\left(\Pi_{k}\right)$ of $\Pi_{k}$ is given by [16]

$$
\tau\left(\Pi_{k}\right)=\sum_{1 \leq i<j \leq k} K\left(e_{i} \wedge e_{j}\right)
$$

The scalar curvature of $\tau(x)$ of $M$ at $x$ is identical with the scalar curvature of the tangent space $T_{x} M$ of $M$ at $x$, that is, $\tau(x)=\tau\left(T_{x} M\right)$. Geometrically, $\tau\left(\Pi_{k}\right)$ is the scalar curvature of the image $\exp _{x}\left(\Pi_{k}\right)$ of $\Pi_{k}$ at $x$ under the exponential map at $x$. If $\Pi_{2}$ is a 2-plane section, $\tau\left(\Pi_{2}\right)$ is simply the sectional curvature $K\left(\Pi_{2}\right)$ of $\Pi_{2},[4,16,17]$.

Now, let us put

$$
h_{i j}^{r}=g\left(h\left(e_{i}, e_{j}\right), e_{r}\right)
$$

where $i, j \in\{1, \ldots, n\}$ and $r \in\{n+1, \ldots, 2 m+1\}$. Then, in view of the equation of Gauss, we have

$$
K\left(e_{i} \wedge e_{j}\right)=\bar{K}\left(e_{i} \wedge e_{j}\right)+\sum_{r=n+1}^{2 m+1}\left(h_{i i}^{r} h_{j j}^{r}-\left(h_{i j}^{r}\right)^{2}\right),
$$

where $K\left(e_{i} \wedge e_{j}\right)$ and $\bar{K}\left(e_{i} \wedge e_{j}\right)$ denote the sectional curvature of the plane section spanned by $e_{i}$ and $e_{j}$ at $x$ in the submanifold $M$ and in the ambient manifold $\bar{M}$, respectively. Taking the summation over the orthonormal frame of the tangent space of $M$ in the above equation, we obtain

$$
2 \tau(x)=2 \bar{\tau}\left(T_{x} M\right)+n^{2}\|H\|^{2}-\|h\|^{2},
$$

where $\bar{\tau}\left(T_{x} M\right)=\sum_{1 \leq i<j \leq n} \bar{K}\left(e_{i} \wedge e_{j}\right)$ denotes the scalar curvature of the $n$-plane section $T_{x} M$ for each $x \in M$ in the ambient manifold $\bar{M}$.

There are different classes of submanifolds which we introduce briefly such as slant submanifolds, CR-submanifolds and semi-slant submanifolds. We shall always consider $\xi$ to be tangent to the submanifold $M$. For a slant submanifold $M$, there is a non-zero vector $X$ tangent to $M$ at $x$ such that $X$ is not proportional to $\xi_{x}$. We denote by $0 \leq \theta(X) \leq \pi / 2$ the angle between $\phi X$ and $T_{x} M$ called the Wirtinger angle. If the Wirtinger angle $\theta(X)$ is constant for all $X \in T_{x} M-\left\langle\xi_{x}\right\rangle$ and $x \in M$, then $M$ is said to be a slant submanifold and the angle $\theta(X)$ is called the slant angle of $M$ [18]. Obviously, if $\theta=0, M$ is invariant and if $\theta=\pi / 2, M$ is an anti-invariant submanifold. A slant submanifold is said to be proper slant if it is neither invariant nor anti-invariant.

We recall the following result for a slant submanifold of an almost contact metric manifold.

Theorem 2.1 [18] Let $M$ be a submanifold of an almost contact metric manifold $\bar{M}$ such that $\xi \in \Gamma(T M)$. Then $M$ is slant if and only if there exists a constant $\lambda \in[0,1]$ such that

$$
P^{2}=\lambda(-I+\eta \otimes \xi)
$$

Furthermore, if $\theta$ is a slant angle, then $\lambda=\cos ^{2} \theta$. 
The following relations are straightforward consequences of equation (2.10)

$$
\begin{aligned}
& g(P X, P Y)=\cos ^{2} \theta(g(X, Y)-\eta(Y) \eta(X)), \\
& g(F X, F Y)=\sin ^{2} \theta(g(X, Y)-\eta(Y) \eta(X))
\end{aligned}
$$

for all $X, Y \in \Gamma(T M)$.

The idea of semi-slant submanifolds of almost Hermitian manifolds was given by Papaghuic [19]. In fact, semi-slant submanifolds were defined on the line of CRsubmanifolds. These submanifolds are defined and investigated by Cabrerizo et al. for almost contact manifolds [20]. They defined these submanifolds as follows.

Definition 2.2 [20, 21] A submanifold $M$ of an almost contact manifold $\bar{M}$ is said to be a semi-slant submanifold if there exist two orthogonal distributions $D$ and $D_{\theta}$ such that:

(i) $T M=D \oplus D_{\theta} \oplus\langle\xi\rangle$.

(ii) $D$ is invariant, i.e., $\phi D \subseteq D$.

(iii) $D_{\theta}$ is a slant distribution with slant angle $\theta \neq \frac{\pi}{2}$.

In the above definition, if $\theta=\pi / 2$ then $M$ is contact CR-submanifold of $\bar{M}$. If $v$ is the invariant subspace of the normal bundle $T^{\perp} M$, then in case of semi-slant submanifolds, the normal bundle $T^{\perp} M$ can be decomposed as follows:

$$
T^{\perp} M=F D_{\theta} \oplus \nu .
$$

For the differential function $\psi$ on $M$, the gradient $\operatorname{grad} \psi$ and the Laplacian $\Delta \psi$ of $\psi$ are defined respectively by

$$
\begin{aligned}
& g(\operatorname{grad} \psi, X)=X \psi, \\
& \Delta \psi=\sum_{i=1}^{n}\left(\left(\nabla_{e_{i}} e_{i}\right) \psi-e_{i} e_{i} \psi\right)
\end{aligned}
$$

for any vector field $X$ tangent to $M$, where $\nabla$ denotes the Riemannian connection on $M$.

\section{Warped product submanifolds}

In this section, we study warped product submanifolds of nearly trans-Sasakian manifolds. We recall the following results on warped products for later use.

Lemma 3.1 Let $M=N_{1} \times_{f} N_{2}$ be a warped product manifold with the warping function $f$. Then

(i) $\nabla_{X} Y \in \Gamma\left(T N_{1}\right)$

(ii) $\nabla_{X} Z=\nabla_{Z} X=(X \ln f) Z$,

(iii) $\nabla_{Z} W=\nabla_{Z}^{N_{2}} W-(g(Z, W) / f) \operatorname{grad} f$

for any $X, Y \in \Gamma\left(T N_{1}\right)$ and $Z, W \in \Gamma\left(T N_{2}\right)$, where $\nabla$ and $\nabla^{N_{2}}$ denote the Levi-Civita connections on $M$ and $N_{2}$, respectively, and $\operatorname{grad} f$ is the gradient off .

Corollary 3.1 On a warped product manifold $M=N_{1} \times_{f} N_{2}$, we have:

(i) $N_{1}$ is totally geodesic in $M$,

(ii) $N_{2}$ is totally umbilical in $M$. 
In the following, we prove the non-existence of warped products of the form $M=N_{1} \times_{f}$ $N_{2}$ in a nearly trans-Sasakian manifold such that $\xi$ is tangent to $N_{2}$.

Theorem 3.1 Let $\bar{M}$ be a nearly trans-Sasakian manifold which is not nearly Sasakian, and let $M=N_{1} \times_{f} N_{2}$ be a warped product submanifold of $\bar{M}$ such that $\xi$ is tangent to $N_{2}$, then $M$ is simply a Riemannian product of $N_{1}$ and $N_{2}$, where $N_{1}$ and $N_{2}$ are any Riemannian submanifolds of $\bar{M}$.

Proof For any $X \in \Gamma\left(T N_{1}\right)$, we have $\left(\bar{\nabla}_{X} \phi\right) \xi+\left(\bar{\nabla}_{\xi} \phi\right) X=-\alpha X-\beta \phi X$. Since for a contact metric manifold $\bar{M},\left(\bar{\nabla}_{\xi} \phi\right) X=0$ [22], hence we get

$$
\phi \bar{\nabla}_{X} \xi=\alpha X+\beta \phi X
$$

Taking the inner product with $\phi X$ in (3.1) and using Lemma 3.1(ii) and the fact that $\xi$ is tangent to $N_{2}$, we get $\beta\|X\|^{2}=0$. This means that the first factor of the warped product vanishes, which proves the theorem completely.

In view of the above theorem, we get a non-existence result about the warped product semi-slant submanifolds in a nearly trans-Sasakian manifold, i.e., there do not exist warped product semi-slant submanifolds $N_{\theta} \times_{f} N_{T}$ and $N_{T} \times_{f} N_{\theta}$ of a nearly trans-Sasakian manifold when the characteristic vector field $\xi$ is a tangent to the second factor. Now, we show that the warped products of type $N_{\theta} \times_{f} N_{T}$ are also Riemannian products if $\xi$ is tangent to the first factor.

Theorem 3.2 There do not exist warped product semi-slant submanifolds of type $M=$ $N_{\theta} \times_{f} N_{T}$ of a nearly trans-Sasakian manifold $\bar{M}$ such that $\xi$ is tangent to $N_{\theta}$, unless $\bar{M}$ is nearly $\beta$-Kenmotsu.

Proof Consider an arbitrary vector $X$ tangent to $N_{T}$, then making use of (1.3) it follows $\left(\bar{\nabla}_{X} \phi\right) \xi+\left(\bar{\nabla}_{\xi} \phi\right) X=-\alpha X-\beta \phi X$. Since $\left(\bar{\nabla}_{\xi} \phi\right) X=0$, for any $X \in \Gamma(T \bar{M})$, thus this relation can be simplified as

$$
-\phi \bar{\nabla}_{X} \xi=-\alpha X-\beta \phi X
$$

Taking the inner product with $X$ in (3.2), we get

$$
g\left(\bar{\nabla}_{X} \xi, \phi X\right)=-\alpha\|X\|^{2} .
$$

By orthogonality of the vector fields $X$ and $\phi X$ and by Lemma 3.1(ii), the left-hand side of (3.3) vanishes identically, hence we reach $\alpha\|X\|^{2}=0$, this means that the first factor of the warped product $N_{\theta} \times_{f} N_{T}$ vanishes, which proves the theorem.

From the above discussion, we conclude that there do not exist warped product semislant submanifolds of type $N_{\theta} \times_{f} N_{T}$ in a nearly trans-Sasakian manifold $\bar{M}$ in both the cases either $\xi$ is tangent to the first factor or to the second. Also, the warped product $N_{T} \times_{f} N_{\theta}$ is just a Riemannian product when the characteristic vector field $\xi$ is tangent to $N_{\theta}$. Now, we discuss the warped product submanifolds $N_{T} \times_{f} N_{\theta}$ such that $\xi$ is tangent to $N_{T}$. 
First, we prove a key lemma characterizing geometric properties of the warped product submanifolds $N_{T} \times_{f} N_{\theta}$ of a nearly trans-Sasakian manifold $\bar{M}$.

Lemma 3.2 Let $M=N_{T} \times_{f} N_{\theta}$ be a warped product semi-slant submanifold of a nearly trans-Sasakian manifold $\bar{M}$ such that $\xi$ is tangent to $N_{T}$. Then the following relations hold:

(i) $\xi \ln f=\beta$,

(ii) $g(h(X, Y), F Z)=0$,

(iii) $g(h(\xi, Z), F W)=-\alpha g(Z, W)$,

(iv) $g(h(X, Z), F Z)=-\{(\phi X \ln f)+\alpha \eta(X)\}\|Z\|^{2}$,

(v) $g(h(X, Z), F P Z)=-g(h(X, P Z), F Z)=\frac{1}{3} \cos ^{2} \theta\{(X \ln f)-\beta \eta(X)\}\|Z\|^{2}$,

(vi) $g(h(X, X), \zeta)=-g(h(\phi X, \phi X), \zeta)$

for any $X, Y \in \Gamma\left(T N_{T}\right)$ and for any $Z, W \in \Gamma\left(T N_{\theta}\right)$ and $\zeta \in \Gamma(v)$.

Proof The first three parts can be proved by the same way as we have proved for contact CR-warped products in [12]. Now, as we consider $\xi$ is tangent to $N_{T}$, then for any $X \in$ $\Gamma\left(T N_{T}\right)$ and $Z \in \Gamma\left(T N_{\theta}\right)$, we have

$$
\left(\bar{\nabla}_{X} \phi\right) Z+\left(\bar{\nabla}_{Z} \phi\right) X=-\alpha \eta(X) Z-\beta \eta(X) \phi Z .
$$

Taking the inner product with $Z$, we obtain

$$
g\left(\left(\bar{\nabla}_{X} \phi\right) Z+\left(\bar{\nabla}_{Z} \phi\right) X, Z\right)=-\alpha \eta(X)\|Z\|^{2} .
$$

Also, we have

$$
\begin{aligned}
\left(\bar{\nabla}_{X} \phi\right) Z & =\bar{\nabla}_{X} \phi Z-\phi \bar{\nabla}_{X} Z \\
& =\nabla_{X} P Z+h(X, P Z)-A_{F Z} X+\nabla_{X}^{\perp} F Z-\phi \nabla_{X} Z-\phi h(X, Z) .
\end{aligned}
$$

Taking the inner product with $Z$ and using Lemma 3.1(ii), we obtain

$$
g\left(\left(\bar{\nabla}_{X} \phi\right) Z, Z\right)=0
$$

Similarly, we can obtain

$$
g\left(\left(\bar{\nabla}_{Z} \phi\right) X, Z\right)=(\phi X \ln f)\|Z\|^{2}+g(h(X, Z), F Z) .
$$

Then from (3.4), (3.5) and (3.6) we obtain part (iv) of the lemma. Now, from the structure equation (1.3) and Lemma 3.1(ii), we have

$$
g\left(\left(\bar{\nabla}_{X} \phi\right) P Z+\left(\bar{\nabla}_{P Z} \phi\right) X, Z\right)=\beta \eta(X) \cos ^{2} \theta\|Z\|^{2}
$$

for any $X \in \Gamma\left(T N_{T}\right)$ and $Z \in \Gamma\left(T N_{\theta}\right)$ such that $\xi$ is tangent to $N_{T}$. Again, by Lemma 3.1(ii) and the Gauss-Weingarten formulas, we obtain

$$
g\left(\left(\bar{\nabla}_{X} \phi\right) P Z, Z\right)=g(h(X, P Z), F Z)-g(h(X, Z), F P Z)
$$

and

$$
g\left(\left(\bar{\nabla}_{P Z} \phi\right) X, Z\right)=g(h(X, P Z), F Z)+(X \ln f) \cos ^{2} \theta\|Z\|^{2} .
$$


Thus from (3.7), (3.8) and (3.9) we derive

$$
2 g(h(X, P Z), F Z)-g(h(X, Z), F P Z)=\{\beta \eta(X)-(X \ln f)\} \cos ^{2} \theta\|Z\|^{2} .
$$

Interchanging $Z$ by $P Z$ in (3.10), we obtain

$$
-2 g(h(X, Z), F P Z)+g(h(X, P Z), F Z)=\{\beta \eta(X)-(X \ln f)\} \cos ^{2} \theta\|Z\|^{2} .
$$

Then, by (3.10) and (3.11), we get

$$
g(h(X, P Z), F Z)=-g(h(X, Z), F P Z),
$$

which is the first equality of the fifth part of the lemma. The second equality of (v) follows from (3.10) and (3.12). For the last part of the lemma, for any $X \in \Gamma\left(T N_{T}\right)$, we have $\bar{\nabla}_{X} \phi X-$ $\phi \bar{\nabla}_{X} X=\alpha\|X\|^{2} \xi-\eta(X) X-\beta \eta(X) \phi X$. By means of (2.1), this relation reduces to

$$
\nabla_{X} \phi X+h(\phi X, X)-\phi \nabla_{X} X-\phi h(X, X)=\alpha\|X\|^{2} \xi-\alpha \eta(X) X-\beta \eta(X) \phi X .
$$

Taking the inner product in the above equation with $\phi \zeta$, for any vector $\zeta \in \Gamma(v)$, we deduce that

$$
g(h(\phi X, X), \phi \zeta)-g(h(X, X), \zeta)=0 .
$$

Interchanging $X$ by $\phi X$ in the above equation and making use of (1.1) and the fact that $v$ is an invariant normal subbundle of $T^{\perp} M$, we have

$$
-g(h(X, \phi X), \phi \zeta)+\eta(X) g(h(\xi, \phi X), \phi \zeta)=g(h(\phi X, \phi X), \zeta) .
$$

Now, by means of (1.3), we derive

$$
h(\phi X, \xi)-2 \phi h(X, \xi)-\phi \nabla_{X} \xi=\alpha(\eta(X) \xi-X)-\beta \phi X .
$$

Taking the inner product with $\phi \zeta$ in (3.15), we obtain

$$
g(h(\phi X, \xi), \phi \zeta)-2 g(h(X, \xi), \zeta)=0 .
$$

Interchanging $\zeta$ by $\phi \zeta$ in the first step and $X$ by $\phi X$ in the second one, taking in consideration that $h(\xi, \xi)=0$, we obtain the following couple of tensorial relations:

$$
g(h(\phi X, \xi), \zeta)+2 g(h(X, \xi), \phi \zeta)=0
$$

and

$$
g(h(X, \xi), \phi \zeta)+2 g(h(\phi X, \xi), \zeta)=0 .
$$

From (3.16) and (3.17) we deduce that

$$
g(h(X, \xi), \phi \zeta)=g(h(\phi X, \xi), \zeta) .
$$


In view of (3.17) and (3.18), we get $g(h(X, \xi), \phi \zeta)=0$. Again, interchanging $X$ by $\phi X$ in this relation yields

$$
g(h(\phi X, \xi), \phi \zeta)=0
$$

Then, by (3.14) and (3.19), we reach

$$
-g(h(\phi X, X), \phi \zeta)-g(h(\phi X, \phi X), \zeta)=0 .
$$

Thus from (3.13) and (3.20) we get the assertion.

\section{An inequality for warped product submanifolds $N_{T} \mathrm{x}_{f} N_{\theta}$}

In the setting of almost contact structures, many authors have proved general inequalities in terms of the squared norm of the second fundamental form and the gradient of the warping function in various structures [12-15]. In fact, all these inequalities are the extension of the original inequality constructed by Chen in the almost Hermitian setting [23]. However, no one proved this relation for warped product semi-slant submanifolds. For this reason, our inequality generalizes the inequalities obtained for CR-warped products in the almost contact setting. Another reason is that a nearly trans-Sasakian structure includes all almost contact structures as a special case.

From now on, we shall follow the following orthonormal basis frame of the ambient manifold $\bar{M}$ for the warped product semi-slant submanifold $M=N_{T} \times_{f} N_{\theta}$ such that $\xi$ is tangent to $N_{T}$. We shall denote by $D$ and $D_{\theta}$ the tangent spaces of $N_{T}$ and $N_{\theta}$, respectively, instead of $T N_{T}$ and $T N_{\theta}$. We set $\left\{e_{1}, \ldots, e_{s}, e_{s+1}=\phi e_{1}, \ldots, e_{\left(n_{1}-1=2 s\right)}=\right.$ $\phi e_{s}, e_{\left(n_{1}=2 s+1\right)}=\xi, e_{n_{1}+1}=e_{1}^{\star}, \ldots, e_{n_{1}+q}=e_{q}^{\star}, e_{n_{1}+q+1}=e_{q+1}^{\star}=\sec \theta P e_{1}^{\star}, \ldots, e_{\left(n=n_{1}+n_{2}\right)}=e_{\left(n_{2}=2 q\right)}^{\star}=$ $\left.\sec \theta P e_{q}^{\star}, e_{n+1}=\csc \theta F e_{1}^{\star}, \ldots, e_{n+n_{2}}=\csc \theta F e_{n_{2}}^{\star}, e_{n+n_{2}+1}=\bar{e}_{1}, \ldots, e_{2 m+1}=\bar{e}_{2 l}\right\}$ as a basis frame of $T \bar{M}$, then $\left\{e_{1}, \ldots, e_{s}, e_{s+1}=\phi e_{1}, \ldots, e_{n_{1}-1}=\phi e_{s}, e_{n_{1}}=\xi, e_{n_{1}+1}=e_{1}^{\star}, \ldots, e_{n_{1}+q}=e_{q}^{\star}, e_{n_{1}+q+1}=\right.$ $\left.e_{q+1}^{\star}=\sec \theta P e_{1}^{\star}, \ldots, e_{\left(n=n_{1}+n_{2}\right)}=e_{\left(n_{2}=2 q\right)}^{\star}=\sec \theta P e_{q}^{\star}\right\}$ are the basis of $T M$ such that $e_{1}, \ldots, e_{s}$, $e_{s+1}=\phi e_{1}, \ldots, e_{n_{1}-1}=\phi e_{s}, e_{n_{1}}=\xi$ are tangent to $D$ and $e_{1}^{\star}, \ldots, e_{q}^{\star}, e_{q+1}^{\star}=\sec \theta P e_{1}^{\star}, \ldots$, $e_{\left(n_{2}=2 q\right)}^{\star}=\sec \theta P e_{q}^{\star}$ are tangent to $D_{\theta}$, hence $\left\{e_{n+1}=\csc \theta F e_{1}^{\star}, \ldots, e_{n+n_{2}}=\csc \theta F e_{n_{2}}^{\star}, e_{n+n_{2}+1}=\right.$ $\left.\bar{e}_{1}, \ldots, e_{2 m+1}=\bar{e}_{2 l}\right\}$ are the basis of the normal bundle $T^{\perp} M$ such that $e_{n+1}=\csc \theta F e_{1}^{\star}, \ldots$, $e_{n+n_{2}}=\csc \theta F e_{n_{2}}^{\star}$ are tangent to $F D_{\theta}$ and $e_{n+n_{2}+1}=\bar{e}_{1}, \ldots, e_{2 m+1}=\bar{e}_{2 l}$ are tangent to the invariant normal subbundle $v$ with dimension $2 l$. We use this frame in the following theorem.

Theorem 4.1 Let $M=N_{T} \times_{f} N_{\theta}$ be a warped product semi-slant submanifold of a nearly trans-Sasakian manifold $\bar{M}$ such that $\xi$ is tangent to $N_{T}$, where $N_{T}$ and $N_{\theta}$ are invariant and proper slant submanifolds of $\bar{M}$ with real dimensions $2 s+1$ and $2 q$, respectively. Then

(i) The second fundamental form $h$ of $M$ satisfies the following inequality:

$$
\|h\|^{2} \geq 2 q\left[\left\{\frac{2}{9} \cot ^{2} \theta+2 \csc ^{2} \theta\right\}\left(\|\operatorname{grad}(\ln f)\|^{2}-\beta^{2}\right)+\alpha^{2}\right] .
$$

(ii) If the equality sign in (i) holds identically, then $N_{T}$ and $N_{\theta}$ are totally geodesic and totally umbilical submanifolds in $\bar{M}$, respectively. 
Proof In view of the adopted frame and the definition of the second fundamental form, it is straightforward to get the following expansion:

$$
\begin{aligned}
\|h\|^{2} & =\sum_{i, j=1}^{n} g\left(h\left(e_{i}, e_{j}\right), h\left(e_{i}, e_{j}\right)\right)=\sum_{r=n+1}^{2 m+1} \sum_{i, j=1}^{n} g\left(h\left(e_{i}, e_{j}\right), e_{r}\right)^{2} \\
& =\sum_{r=n+1}^{n+n_{2}} \sum_{i, j=1}^{n} g\left(h\left(e_{i}, e_{j}\right), e_{r}\right)^{2}+\sum_{r=n+n_{2}+1}^{2 m+1} \sum_{i, j=1}^{n} g\left(h\left(e_{i}, e_{j}\right), e_{r}\right)^{2} \\
& \geq \sum_{r=n+1}^{n+n_{2}} \sum_{i, j=1}^{n} g\left(h\left(e_{i}, e_{j}\right), e_{r}\right)^{2}=\sum_{l=2 s+2}^{n} \sum_{i, j=1}^{n} g\left(h\left(e_{i}, e_{j}\right), \phi e_{l}\right)^{2} .
\end{aligned}
$$

Using the orthonormal frame of $D$ and $D_{\theta}$ gives

$$
\begin{aligned}
\|h\|^{2} \geq & \sum_{l=2 s+2}^{n} \sum_{i, j=1}^{2 s+1} g\left(h\left(e_{i}, e_{j}\right), \phi e_{l}\right)^{2}+2 \sum_{j, l=2 s+2}^{n} \sum_{i=1}^{2 s+1} g\left(h\left(e_{i}, e_{j}\right), \phi e_{l}\right)^{2} \\
& +\sum_{i, j, l=2 s+2}^{n} g\left(h\left(e_{i}, e_{j}\right), \phi e_{l}\right)^{2} .
\end{aligned}
$$

By Lemma 3.2(ii), the first term of the right-hand side in (4.2) is identically zero, so let us compute the next term

$$
\|h\|^{2} \geq 2 \sum_{j, l=2 s+2}^{n} \sum_{i=1}^{2 s} g\left(h\left(e_{i}, e_{j}\right), \phi e_{l}\right)^{2}+2 \sum_{j, l=2 s+2}^{n} g\left(h\left(\xi, e_{j}\right), \phi e_{l}\right)^{2} .
$$

Making use of Lemma 3.2(iii), the second term of the right-hand side in (4.3) can be evaluated, while by means of the orthonormal frame the first term is expanded to give four terms; as a result (4.3) takes the following form:

$$
\begin{aligned}
\|h\|^{2} \geq & 2 \csc ^{2} \theta \sum_{j=1}^{q} \sum_{i=1}^{2 s} g\left(h\left(e_{i}, e_{j}\right), F e_{j}^{\star}\right)^{2} \\
& +2 \csc ^{2} \theta \sec ^{2} \theta \sum_{j=1}^{q} \sum_{i=1}^{2 s} g\left(h\left(e_{i}, P e_{j}^{\star}\right), F e_{j}^{\star}\right)^{2} \\
& +2 \csc ^{2} \theta \sec ^{2} \theta \sum_{j=1}^{q} \sum_{i=1}^{2 s} g\left(h\left(e_{i}, e_{j}\right), F P e_{j}^{\star}\right)^{2} \\
& +2 \csc ^{2} \theta \sec ^{4} \theta \sum_{j=1}^{q} \sum_{i=1}^{2 s} g\left(h\left(e_{i}, P e_{j}^{\star}\right), F P e_{j}^{\star}\right)^{2}+2 \sum_{j, l=2 s+2}^{n}\left(-\alpha g\left(e_{j}, e_{l}\right)\right)^{2} .
\end{aligned}
$$

Using Lemma 3.2(iii)-(v), we derive

$$
\begin{aligned}
\|h\|^{2} \geq & 2 \csc ^{2} \theta \sum_{j=1}^{q} \sum_{i=1}^{2 s}\left(\left(\phi e_{i} \ln f\right)+\alpha \eta\left(e_{i}\right)\right)^{2}\left\|e_{j}\right\|^{4} \\
& +\frac{2}{9} \cos ^{2} \theta \csc ^{2} \theta \sum_{j=1}^{q} \sum_{i=1}^{2 s}\left(\left(e_{i} \ln f\right)-\beta \eta\left(e_{i}\right)\right)^{2}\left\|e_{j}\right\|^{4}
\end{aligned}
$$




$$
\begin{aligned}
& +\frac{2}{9} \cos ^{2} \theta \csc ^{2} \theta \sum_{j=1}^{q} \sum_{i=1}^{2 s}\left(\left(e_{i} \ln f\right)-\beta \eta\left(e_{i}\right)\right)^{2}\left\|e_{j}\right\|^{4} \\
& +2 \csc ^{2} \theta \sum_{j=1}^{q} \sum_{i=1}^{2 s}\left(\left(\phi e_{i} \ln f\right)+\alpha \eta\left(e_{i}\right)^{2}\right)\left\|e_{j}\right\|^{4}+2 q \alpha^{2} .
\end{aligned}
$$

In view of the assumed orthonormal frame, the 1-form $\eta\left(e_{i}\right)$ is identically zero for all $i \in$ $\{1, \ldots, 2 s\}$, hence we reach

$$
\begin{aligned}
\|h\|^{2} \geq & 4 \csc ^{2} \theta \sum_{j=1}^{q} \sum_{i=1}^{2 s}\left(\phi e_{i} \ln f\right)^{2}\left\|e_{j}\right\|^{4} \\
& +\frac{4}{9} \csc ^{2} \theta \cos ^{2} \theta \sum_{j=1}^{q} \sum_{i=1}^{2 s}\left(e_{i} \ln f\right)^{2}\left\|e_{j}\right\|^{4}+2 q \alpha^{2}
\end{aligned}
$$

Then from (2.14) and Lemma 3.2(i) the above inequality takes the form

$$
\|h\|^{2} \geq 2 q\left[\left\{\frac{2}{9} \cot ^{2} \theta+2 \csc ^{2} \theta\right\}\left(\|\nabla \ln f\|^{2}-\beta^{2}\right)+\alpha^{2}\right],
$$

which is the inequality (i). Now, assume that the equality sign in (4.1) holds identically, then from (4.2), (4.3) and Lemma 3.2(ii) we deduce that

$$
h(D, D)=0, \quad h\left(D_{\theta}, D_{\theta}\right)=0, \quad h\left(D, D_{\theta}\right) \subset F D_{\theta} .
$$

Hence, combining statement of Corollary 3.1(i) with the first condition in (4.7) shows that $N_{T}$ is totally geodesic in $\bar{M}$. On the other hand, if we denote by $h^{\theta}$ the second fundamental form of $N_{\theta}$ in $M$, then we get

$$
g\left(h^{\theta}(Z, W), X\right)=g\left(\nabla_{Z} W, X\right)=-(X \ln f) g(Z, W)=-g(Z, W) g(\nabla \ln f, X),
$$

which is equivalent to

$$
h^{\theta}(Z, W)=-\nabla \ln f g(Z, W) .
$$

This means that $N_{\theta}$ is totally umbilical in $M$, thus the second condition of (4.7) with (4.8) and Corollary 3.1(ii) imply that $N_{\theta}$ is totally umbilical in $\bar{M}$. Also, all three conditions of (4.7) give the minimality of $M$.

Note In inequality (5.1), if $\alpha=0$ and $\beta=1$, then it reduces to

$$
\|h\|^{2} \geq 2 q\left[\left\{\frac{2}{9} \cot ^{2} \theta+2 \csc ^{2} \theta\right\}\left(\|\nabla \ln f\|^{2}-1\right)\right]
$$

which is the inequality for nearly Kenmotsu manifolds. Also, if $\alpha=1$ and $\beta=0$, then the inequality reduces for the nearly Sasakian manifolds. The equality cases can also be discussed. 
Remark 1 Theorem 3.1 in [13], Theorem 3.4 in [14] and Theorem 3.2 in [15] are the special cases of the above inequality.

Remark 2 The above inequality generalizes Theorem 4.1 in [12].

\section{Another inequality for warped products}

Let $\varphi: M=N_{1} \times_{f} N_{2} \longrightarrow \bar{M}$ be an isometric immersion of the warped product $N_{1} \times_{f} N_{2}$ into the Riemannian manifold $\bar{M}$ of constant sectional curvature $c$. Denote by $n_{1}, n_{2}, n$ the dimensions of $N_{1}, N_{2}, N_{1} \times_{f} N_{2}$, respectively. Then for unit vector fields $X, Z$ tangent to $N_{1}, N_{2}$, respectively, we have

$$
K(X \wedge Z)=g\left(\nabla_{Z} \nabla_{X} X-\nabla_{X} \nabla_{Z} X, Z\right)=(1 / f)\left\{\left(\nabla_{X} X\right) f-X^{2} f\right\} .
$$

If we choose the local orthonormal frame $e_{1}, \ldots, e_{n}$ such that $e_{1}, \ldots, e_{n_{1}}$ are tangent to $N_{1}$ and $e_{n_{1}+1}, \ldots, e_{n}$ are tangent to $N_{2}$, then we have

$$
\frac{\Delta f}{f}=\sum_{i=1}^{n_{1}} K\left(e_{i} \wedge e_{j}\right)
$$

for each $j=n_{1}+1, \ldots, n$.

In this section, our aim is to develop a new method which is giving a useful formula for the squared norm of the mean curvature vector $\vec{H}$ under $\varphi$. Geometrically, this formula declares the $N_{T}$-minimality of $\varphi$.

We know that

$$
\|H\|^{2}=\frac{1}{n^{2}} \sum_{r=n+1}^{2 m+1}\left(h_{11}^{r}+\cdots+h_{n n}^{r}\right)^{2} .
$$

Taking in consideration that $\left(n=n_{1}+n_{2}\right)$, where $n_{1}$ and $n_{2}$ are the dimensions of $N_{T}$ and $N_{\theta}$, respectively, we obtain

$$
\|H\|^{2}=\frac{1}{n^{2}} \sum_{r=n+1}^{2 m+1}\left(h_{11}^{r}+\cdots+h_{n_{1} n_{1}}^{r}+h_{n_{1}+1 n_{1}+1}^{r}+\cdots+h_{n n}^{r}\right)^{2} .
$$

Moreover, for every $r \in\{n+1, \ldots, 2 m+1\}$, using the frame of $D$ and the fact that $h(\xi, \xi)=0$, then $n_{1}$ coefficients of the right-hand side can be decomposed as follows:

$$
\begin{aligned}
& \left(h_{11}^{r}+\cdots+h_{n_{1} n_{1}}^{r}+h_{n_{1}+1 n_{1}+1}^{r}+\cdots+h_{n n}^{r}\right)^{2} \\
& \quad=\left(h_{11}^{r}+\cdots+h_{s s}^{r}+h_{s+1 s+1}^{r}+\cdots+h_{2 s 2 s}^{r}+h_{\xi \xi}^{r}+h_{n_{1}+1 n_{1}+1}^{r}+\cdots+h_{n n}^{r}\right)^{2} \\
& =\left(h_{11}^{r}+\cdots+h_{s s}^{r}+h_{s+1 s+1}^{r}+\cdots+h_{2 s 2 s}^{r}+h_{n_{1}+1 n_{1}+1}^{r}+\cdots+h_{n n}^{r}\right)^{2} .
\end{aligned}
$$

From (2.7) we know that $e_{r}$ belongs to the normal bundle $T M^{\perp}$ for every $r \in\{n+1, \ldots$, $2 m+1\}$. Then in view of (2.13) we have two cases: either it belongs to $F D_{\theta}$ or to $v$.

Case (i). If $e_{r} \in \Gamma\left(F D_{\theta}\right)$, then from Lemma 3.2(ii) we know that $g(h(X, X), F Z)=0$ for any $X \in \Gamma(D)$ and $Z \in \Gamma\left(D_{\theta}\right)$; consequently (5.3) reduces to

$$
\left(h_{11}^{r}+\cdots+h_{n_{1} n_{1}}^{r}+h_{n_{1}+1 n_{1}+1}^{r}+\cdots+h_{n n}^{r}\right)^{2}=\left(h_{n_{1}+1 n_{1}+1}^{r}+\cdots+h_{n n}^{r}\right)^{2} .
$$


Case (ii). If $e_{r} \in \Gamma(v)$, then by means of Lemma 3.2(vi), we can make an expansion of (5.3) as follows:

$$
\begin{aligned}
\left(h_{11}^{r}\right. & \left.+\cdots+h_{n_{1} n_{1}}^{r}+h_{n_{1}+1 n_{1}+1}^{r}+\cdots+h_{n n}^{r}\right)^{2} \\
= & \left(g\left(h\left(e_{1}, e_{1}\right), e_{r}\right)+\cdots+g\left(h\left(e_{s}, e_{s}\right), e_{r}\right)+g\left(h\left(\phi e_{1}, \phi e_{1}\right), e_{r}\right)\right. \\
& \left.+\cdots+g\left(h\left(\phi e_{s}, \phi e_{s}\right), e_{r}\right)+h_{n_{1}+1 n_{1}+1}^{r}+\cdots+h_{n n}^{r}\right)^{2} \\
= & \left(g\left(h\left(e_{1}, e_{1}\right), e_{r}\right)+\cdots+g\left(h\left(e_{s}, e_{s}\right), e_{r}\right)-g\left(h\left(e_{1}, e_{1}\right), e_{r}\right)-\cdots\right. \\
& \left.\quad-g\left(h\left(e_{s}, e_{s}\right), e_{r}\right)+h_{n_{1}+1 n_{1}+1}^{r}+\cdots+h_{n n}^{r}\right)^{2} \\
= & \left(h_{n_{1}+1 n_{1}+1}^{r}+\cdots+h_{n n}^{r}\right)^{2} .
\end{aligned}
$$

Then from (5.4) and (5.5) we can deduce that

$$
\left(h_{11}^{r}+\cdots+h_{n_{1} n_{1}}^{r}+h_{n_{1}+1 n_{1}+1}^{r}+\cdots+h_{n n}^{r}\right)^{2}=\left(h_{n_{1}+1 n_{1}+1}^{r}+\cdots+h_{n n}^{r}\right)^{2}
$$

for every normal vector $e_{r}$ belongs to the normal bundle $T^{\perp} M$. In other words,

$$
\sum_{r=n+1}^{2 m+1}\left(h_{11}^{r}+\cdots+h_{n_{1} n_{1}}^{r}+h_{n_{1}+1 n_{1}+1}^{r}+\cdots+h_{n n}^{r}\right)^{2}=\sum_{r=n+1}^{2 m+1}\left(h_{n_{1}+1 n_{1}+1}^{r}+\cdots+h_{n n}^{r}\right)^{2} .
$$

By the end of this discussion, we can state the following lemma.

Lemma 5.1 Let $\varphi: M=N_{T} \times_{f} N_{\theta} \longrightarrow \bar{M}$ be an isometric immersion from a warped product semi-slant submanifold into a nearly trans-Sasakian manifold $\bar{M}$. Then we have

$$
\|\vec{H}\|^{2}=\frac{1}{n^{2}} \sum_{r=n+1}^{2 m+1}\left(h_{n_{1}+1 n_{1}+1}^{r}+\cdots+h_{n n}^{r}\right)^{2}
$$

i.e., $\varphi$ is an $N_{T}$-minimal immersion, where $\vec{H}$ is the mean curvature vector and $n_{1}, n_{2}, n$ and $(2 m+1)$ are the dimensions of $N_{T}, N_{\theta}, M$ and $\bar{M}$, respectively.

From the Gauss equation and the above key Lemma 5.1, we are able to state and prove the following general inequality.

Theorem 5.1 Let $\varphi: M=N_{T} \times_{f} N_{\theta} \longrightarrow \bar{M}$ be an isometric immersion from a warped product semi-slant submanifold into a nearly trans-Sasakian manifold $\bar{M}$ such that $\xi$ is tangent to $N_{T}$. Then we have

(i) $\frac{1}{2}\|h\|^{2} \geq \bar{\tau}(T M)-\bar{\tau}\left(T N_{T}\right)-\bar{\tau}\left(T N_{\theta}\right)-\frac{n_{2} \Delta f}{f}$, where $n_{2}$ is the dimension of $N_{\theta}$.

(ii) If the equality sign in (i) holds identically, then $N_{T}$ and $N_{\theta}$ are totally geodesic and totally umbilical submanifolds in $\bar{M}$, respectively.

Proof We start by recalling (2.9) as a consequence of (2.5) as

$$
\|h\|^{2}=-2 \tau+2 \bar{\tau}(T M)+n^{2}\|H\|^{2} .
$$


Making use of (2.6) in the above equation, we deduce

$$
\|h\|^{2}=-2 \sum_{i=1}^{n_{1}} \sum_{j=n_{1}+1}^{n} K\left(e_{i} \wedge e_{j}\right)-2 \tau\left(T N_{T}\right)-2 \tau\left(T N_{\theta}\right)+2 \bar{\tau}(T M)+n^{2}\|H\|^{2} .
$$

Then from Lemma 3.2 and relation (2.8) it follows

$$
\begin{aligned}
\|h\|^{2}= & -\frac{2 n_{2} \Delta f}{f}-2 \bar{\tau}\left(T N_{T}\right)-2 \sum_{r=n+1}^{2 m+1} \sum_{1 \leq i<k \leq n_{1}}\left(h_{i i}^{r} h_{k k}^{r}-\left(h_{i k}^{r}\right)^{2}\right)-2 \bar{\tau}\left(T N_{\theta}\right) \\
& -2 \sum_{r=n+1}^{2 m+1} \sum_{n_{1}+1 \leq j<t \leq n}\left(h_{j j}^{r} h_{t t}^{r}-\left(h_{j t}^{r}\right)^{2}\right)+2 \bar{\tau}(T M)+n^{2}\|H\|^{2} .
\end{aligned}
$$

The above equation is equivalent to the following form:

$$
\begin{aligned}
\|h\|^{2}= & -\frac{2 n_{2} \Delta f}{f}-2 \bar{\tau}\left(T N_{T}\right)-\sum_{r=n+1}^{2 m+1} \sum_{1 \leq i \neq k \leq n_{1}}\left(h_{i i}^{r} h_{k k}^{r}-\left(h_{i k}^{r}\right)^{2}\right)-2 \bar{\tau}\left(T N_{\theta}\right) \\
& +2 \bar{\tau}(T M)-\sum_{r=n+1}^{2 m+1} \sum_{n_{1}+1 \leq j \neq t \leq n}\left(h_{j j}^{r} h_{t t}^{r}-\left(h_{j t}^{r}\right)^{2}\right)+n^{2}\|H\|^{2} .
\end{aligned}
$$

The above equation takes the following form when we add and subtract the same term on the right-hand side:

$$
\begin{aligned}
\|h\|^{2}= & -\frac{2 n_{2} \Delta f}{f}-2 \bar{\tau}\left(T N_{T}\right)-\sum_{r=n+1}^{2 m+1}\left(\left(h_{11}^{r}\right)^{2}+\cdots+\left(h_{n_{1} n_{1}}^{r}\right)^{2}\right) \\
& -\sum_{r=n+1}^{2 m+1} \sum_{1 \leq i \neq k \leq n_{1}}\left(h_{i i}^{r} h_{k k}^{r}-\left(h_{i k}^{r}\right)^{2}\right)+\sum_{r=n+1}^{2 m+1}\left(\left(h_{11}^{r}\right)^{2}+\cdots+\left(h_{n_{1} n_{1}}^{r}\right)^{2}\right) \\
& -2 \bar{\tau}\left(T N_{\theta}\right)-\sum_{r=n+1}^{2 m+1} \sum_{n_{1}+1 \leq j \neq t \leq n}\left(h_{j j}^{r} h_{t t}^{r}-\left(h_{j t}^{r}\right)^{2}\right)+2 \bar{\tau}(T M)+n^{2}\|H\|^{2} \\
= & -\frac{2 n_{2} \Delta f}{f}-2 \bar{\tau}\left(T N_{T}\right)+\sum_{r=n+1}^{2 m+1} \sum_{i, k=1}^{n_{1}}\left(h_{i k}^{r}\right)^{2}-\sum_{r=n+1}^{2 m+1}\left(h_{11}^{r}+\cdots+h_{n_{1} n_{1}}^{r}\right)^{2} \\
& -2 \bar{\tau}\left(T N_{\theta}\right)-\sum_{r=n+1}^{2 m+1} \sum_{n_{1}+1 \leq j \neq t \leq n}\left(h_{j j}^{r} h_{t t}^{r}-\left(h_{j t}^{r}\right)^{2}\right)+2 \bar{\tau}(T M)+n^{2}\|H\|^{2} .
\end{aligned}
$$

Similarly, we can add and subtract the same term for the sixth term in the above equation; and finally, we derive

$$
\begin{aligned}
\|h\|^{2}= & -\frac{2 n_{2} \Delta f}{f}+2 \bar{\tau}(T M)-2 \bar{\tau}\left(T N_{T}\right)+\sum_{r=n+1}^{2 m+1} \sum_{i, k=1}^{n_{1}}\left(h_{i k}^{r}\right)^{2} \\
& +\sum_{r=n+1}^{2 m+1} \sum_{j, t=n_{1}+1}^{n}\left(h_{j t}^{r}\right)^{2}-\sum_{r=n+1}^{2 m+1}\left(h_{11}^{r}+\cdots+h_{n_{1} n_{1}}^{r}\right)^{2}-2 \bar{\tau}\left(T N_{\theta}\right) \\
& -\sum_{r=n+1}^{2 m+1}\left(h_{n_{1}+1 n_{1}+1}^{r}+\cdots+h_{n n}^{r}\right)^{2}+n^{2}\|H\|^{2} .
\end{aligned}
$$


Taking account of Lemma 5.1, we get the inequality (i). For the equality case, from the last relation we get

$$
\sum_{r=n+1}^{2 m+1} \sum_{i, k=1}^{n_{1}} g\left(h\left(e_{i}, e_{k}\right), e_{r}\right)=0
$$

and

$$
\sum_{r=n+1}^{2 m+1} \sum_{j, t=n_{1}+1}^{n} g\left(h\left(e_{j}, e_{t}\right), e_{r}\right)=0
$$

From (5.6) and (5.7) we obtain that the immersion $\varphi: M \rightarrow \bar{M}$ is totally geodesic. Also, from Corollary 3.1 we know that the immersion $N_{T} \rightarrow M$ is totally geodesic and the immersion $N_{\theta} \rightarrow M$ is totally umbilical, hence the result (ii).

\section{Competing interests}

The authors declare that they have no competing interests.

\section{Authors' contributions}

All authors contributed equally to the writing of this paper. All authors read and approved the final manuscript.

\section{Acknowledgements}

The authors would like to express their hearty thanks to anonymous referees for their valuable suggestions and comments. The second author is supported by the Research Grant RG278-14AFR, University of Malaya.

\section{Received: 17 December 2013 Accepted: 3 July 2014 Published: 04 Sep 2014}

\section{References}

1. Nolker, S: Isometric immersions of warped products. Differ. Geom. Appl. 6, 1-30 (1996)

2. O'Neill, B: Semi-Riemannian Geometry with Applications to Relativity. Pure and Applied Mathematics, vol. 103. Academic Press, New York (1983)

3. Bishop, RL, O'Neill, B: Manifolds of negative curvature. Trans. Am. Math. Soc. 145, 1-49 (1969)

4. Chen, BY: Geometry of warped products as Riemannian submanifolds and related problems. Soochow J. Math. 28 , 125-156 (2002)

5. Uddin, S, Khan, VA, Khan, KA: Warped product submanifolds of a Kenmotsu manifold. Turk. J. Math. 36, $319-330$ (2012)

6. Blair, DE: Contact Manifolds in Riemannian Geometry. Lecture Notes in Math., vol. 509. Springer, Berlin (1976)

7. Blair, DE, Showers, DK: Almost contact manifolds with killing structures tensors. II. J. Differ. Geom. 9, 577-582 (1974)

8. Chinea, D, Gonzalez, C: A classification of almost contact metric manifolds. Ann. Mat. Pura Appl. 156, 15-36 (1990)

9. Gherghe, C: Harmonicity of nearly trans-Sasakian manifolds. Demonstr. Math. 33, 151-157 (2000)

10. Kim, JS, Ximin, L, Tripathi, MM: On semi-invariant submanifolds of nearly trans-Sasakian manifold. Int. J. Pure Appl. Math. Sci. 1, 15-34 (2004)

11. Al-Solamy, FR, Khan, VA: A note on slant submanifolds of nearly trans-Sasakian manifolds. Math. Slovaca 60, 129-136 (2010)

12. Mustafa, A, Uddin, S, Khan, VA, Wong, BR: Contact CR-warped product submanifolds of nearly trans-Sasakian manifolds. Taiwan. J. Math. 17, 1473-1486 (2013)

13. Kadri, A, Ridvan, E, Mihai, I, Murathan, C: Contact CR-warped product submanifolds in Kenmotsu space forms. J. Korean Math. Soc. 42, 1101-1110 (2005)

14. Munteanu, MI: Warped product contact CR-submanifolds of Sasakian space forms. Publ. Math. (Debr.) 66, 75-120 (2005)

15. Uddin, S, Khan, KA: An inequality for contact CR-warped product submanifolds of nearly cosymplectic manifolds J. Inequal. Appl. 2012, 304 (2012)

16. Chen, BY: On isometric minimal immersions from warped products into real space forms. Proc. Edinb. Math. Soc. 45 579-587 (2002)

17. Chen, BY: On warped product immersions. J. Geom. 82, 36-49 (2005)

18. Cabrerizo, JL, Carriazo, A, Fernandez, LM, Fernandez, M: Slant submanifolds in Sasakian manifolds. Glasg. Math. J. 42, 125-138 (2000)

19. Papaghuic, N: Semi-slant submanifolds of a Kaehlerian manifold. An. Ştiinţ. Univ. 'Al.I. Cuza' laşi, Mat. 40, 55-61 (1994)

20. Carriazo, A: New Developments in Slant Submanifolds Theory. Narosa Publishing House, New Delhi (2002)

21. Cabrerizo, JL, Carriazo, A, Fernandez, LM, Fernandez, M: Semi-slant submanifolds of a Sasakian manifold. Geom. Dedic. 78, 183-199 (1999)

22. Blair, DE: Almost contact manifolds with killing structure tensors. I. Pac. J. Math. 39, 285-292 (1971)

23. Chen, BY: Geometry of warped product CR-submanifolds in Kaehler manifolds II. Monatshefte Math. 134, 103-119 (2001) 
10.1186/1029-242X-2014-346

Cite this article as: Mustafa et al.: Generalized inequalities on warped product submanifolds in nearly trans-Sasakian manifolds. Journal of Inequalities and Applications 2014, 2014:346

Submit your manuscript to a SpringerOpen ${ }^{\circ}$ journal and benefit from:

- Convenient online submission

- Rigorous peer review

- Immediate publication on acceptance

Open access: articles freely available online

- High visibility within the field

- Retaining the copyright to your article

Submit your next manuscript at $\boldsymbol{s p r i n g e r o p e n . c o m ~}$ 